\title{
A population-based study of the frequency and predictors of induced abortion among women with schizophrenia*
}

\author{
Hilary K. Brown, Cindy-Lee Dennis, Paul Kurdyak and Simone N. Vigod
}

\section{Background}

Induced abortion is an indicator of access to, and quality of reproductive healthcare, but rates are relatively unknown in women with schizophrenia.

\begin{abstract}
Aims
We examined whether women with schizophrenia experience increased induced abortion compared with those without schizophrenia, and identified factors associated with induced abortion risk.
\end{abstract}

\section{Method}

In a population-based, repeated cross-sectional study (20112013), we compared women with and without schizophrenia in Ontario, Canada on rates of induced abortions per 1000 women and per 1000 live births. We then followed a longitudinal cohort of women with schizophrenia aged $15-44$ years $(n=11149)$ from 2011, using modified Poisson regression to identify risk factors for induced abortion.

\section{Results}

Women with schizophrenia had higher abortion rates than those without schizophrenia in all years (15.5-17.5 v. 12.8-13.6 per 1000 women; largest rate ratio, $1.33 ; 95 \% \mathrm{Cl} 1.16-1.54)$. They also had higher abortion ratios (592-736 v. 321-341 per 1000 live births; largest rate ratio, 2.25; 95\% $\mathrm{Cl} 1.96-2.59)$. Younger age (<25 years; adjusted relative risk (aRR), 1.84; 95\% Cl 1.39-2.44), multiparity (aRR 2.17, 95\% Cl 1.66-2.83), comorbid non-psychotic mental illness (aRR 2.15, 95\% Cl 1.34-3.46) and substance misuse disorders (aRR 1.85, 95\% Cl 1.47-2.34) were associated with increased abortion risk.

\section{Conclusions}

These results demonstrate vulnerability related to reproductive healthcare for women with schizophrenia. Evidence-based interventions to support optimal sexual health, particularly in young women, those with psychiatric and addiction comorbidity, and women who have already had a child, are warranted.

\section{Declaration of interest}

None.

\section{Keywords}

Schizophrenia; induced abortion; population-based study.

\section{Copyright and usage}

(c) The Royal College of Psychiatrists 2018
Schizophrenia is a chronic psychotic disorder characterised by positive symptoms such as delusions and deficits in emotional reactivity and social functioning. ${ }^{1}$ Because of hyperprolactinemia associated with first-generation antipsychotics and segregation of many women with schizophrenia in institutions, childbearing rates in this population were low historically. ${ }^{2}$ With introduction of community-based care and fertility-sparing second-generation antipsychotics, women with schizophrenia are now increasingly experiencing pregnancy. ${ }^{2}$ However, their reproductive health is incompletely understood. Induced abortion, defined as a pregnancy termination that is carried out by medication or surgery, is a key indicator of access to, and quality of reproductive healthcare. ${ }^{3}$ Several small clinical studies and commentaries have suggested that women with schizophrenia may have high rates of induced abortions. ${ }^{4,5}$ However, epidemiological evidence is less certain. A Finnish study found that the rate of induced abortion per 1000 follow-up years did not differ between 1587 women with schizophrenia and 7765 age- and place of birth-matched controls without schizophrenia (22.9 v. 24.9 per 1000 follow-up years). ${ }^{6}$ However, in those with a pregnancy, women with schizophrenia were more likely to terminate their pregnancies $(59.1 \%$ v. $25.9 \%$; adjusted risk ratio, 2.28; 95\% CI 2.20-2.36). ${ }^{6}$ A Danish study of women with no previous pregnancies found that the incidence of induced abortion was lower in women with schizophrenia than in those without any mental illness (incidence rate ratio, 0.90; 95\% CI $0.81-0.99) .^{7}$ Given the heterogeneity of previous studies'

* Previously presented as a poster at the Canadian National Perinatal Research Meeting in Banff, Alberta, Canada, on 14 February 2018. methods and findings, it is difficult to make definitive conclusions about induced abortion risk in women with schizophrenia. To our knowledge, there are no North American studies on this topic, and no population-based studies exploring risk factors for induced abortion in this vulnerable population.

We aimed to compare the risk for induced abortion in women with and without schizophrenia in the entire population of Ontario, Canada, and to examine risk factors for induced abortion in women with schizophrenia.

\section{Method}

\section{Study design and setting}

We conducted a population-based study in Ontario, Canada. Ontario is Canada's largest province, with 13.6 million residents. All Ontario residents receive publicly funded healthcare, which covers medically necessary physician and hospital services, including medically and surgically induced abortion, at no cost to the patient. Repeated cross-sectional samples of women with and without schizophrenia aged 15-44 years at the mid-points of the 2011, 2012 and 2013 fiscal years were studied to generate abortion rates (annual number of induced abortions per 1000 reproductive-aged women) and abortion ratios (annual number of induced abortions per 1000 live births). ${ }^{3}$ A cohort of women with schizophrenia aged 15-44 years was then followed from 2011 to 2013 to generate risk factors for induced abortion within this group. The use of data in this project was authorised under section 45 of 
Ontario's Personal Health Information Protection Act, which does not require review by a Research Ethics Board.

\section{Data sources}

Health administrative data resulting from healthcare encounters of all Ontario residents were accessed and analysed at the Institute for Clinical Evaluative Sciences (Toronto, Canada). We used the Registered Persons Database to obtain birth date, postal code and date of death; the Ontario Health Insurance Plan database to obtain out-patient physician visit data; the National Ambulatory Care Reporting System to obtain emergency department visit data; the Canadian Institute for Health Information Discharge Abstract Database to obtain hospital admission and in-patient data and the Ontario Mental Health Reporting System to obtain psychiatric hospital admission and in-patient data. Individuallevel data were linked deterministically across databases by a unique encoded identifier. Physician visit data are recorded using physician billing claim codes, hospital data are recorded using the Canadian Coding Standards for the ICD-10 (after 2002) ${ }^{8}$ and the Canadian Classification of Health Interventions, and psychiatric hospital admission and in-patient data are recorded using the DSM-IV. ${ }^{9}$ Sociodemographic data, physician billing claims and primary diagnoses in hospital databases have been shown to be complete, valid and reliable. ${ }^{10}$

\section{Exposure}

Women with schizophrenia were those with two or more physician visits or one or more admissions to hospital for schizophrenia, schizoaffective disorder or psychotic disorder not otherwise specified in the 2 years before each successive 12-month period (2011, 2012 and 2013), to reflect active disease (Ontario Health Insurance Plan billing codes: 295, 298; ICD-10 codes: F20, F25, F29; DSM-IV: schizophrenia, schizoaffective disorder). ${ }^{11}$ Women with psychotic disorder not otherwise specified were included in this definition because the majority go on to receive a diagnosis of schizophrenia. ${ }^{12}$ This algorithm has a sensitivity of $93.9 \%$ and specificity of $50.0 \%$ compared with clinical charts. ${ }^{11}$ The comparison group comprised women without schizophrenia. Women with intellectual disability, genetic conditions associated with intellectual disability such as Down syndrome, and developmental disabilities such as autism were excluded from the cohort altogether because these conditions are more common among women with schizophrenia and may be associated with their own unique risks for induced abortion. ${ }^{13}$

\section{Outcomes}

The primary outcomes were the general abortion rate and abortion ratio. The most common way to measure induced abortion in the population is to calculate the abortion rate per 1000 reproductiveaged women. ${ }^{3}$ Although this provides an indication of the frequency of induced abortion in specific populations, it does not inform us about the occurrence of induced abortion after taking into account the underlying birth rate. ${ }^{3}$ As such, the abortion ratio, defined as the number of induced abortions per 1000 live births, is often also reported. ${ }^{3}$ The abortion rate herein was defined as the number of induced abortions per 1000 women aged 15-44 years. ${ }^{3}$ The abortion ratio was defined as the ratio of induced abortions per 1000 live births among women aged $15-44$ years. ${ }^{3}$ We also examined age-specific abortion rates and abortion ratios, calculated for 5-year age groups: 15-19, 20-24, 25-29, 30-34, 35-39 and 40-44 years. Induced abortions included medical and surgical abortions identified in physicians' offices and clinics (Ontario Health Insurance Plan billing codes: 635 or 895 and S752; or 635 or 895 and S785, A920 or P001) as well as hospitals (ICD-10 codes: O04 or O08 and CCI: 5CA20FK, 5CA24, 5CA88, FCA89 (INATSTAT not equal to A)). ${ }^{14}$ Although validation data are not available for this algorithm, hospital-based induced abortion data are expected to be complete and accurate because of mandatory reporting requirements. ${ }^{15}$

\section{Covariates}

Covariates were age, parity, neighbourhood income quintile, region of residence, severity of schizophrenia, stable and unstable chronic medical conditions, comorbid non-psychotic mental illness, substance misuse disorders and continuity of primary care. Neighbourhood income quintile and region of residence were identified by linking residential postal code with census information; rural residences were in communities with a population of $<10000$ residents. Severity of schizophrenia was defined by the number of admissions to hospital for schizophrenia, schizoaffective disorder or psychotic disorder not otherwise specified in the 2 years before the index date. Chronic medical conditions were classified using the Johns Hopkins ACG ${ }^{\oplus}$ System Version 9.0 collapsed ambulatory diagnostic groups; stability was determined on the basis of disease severity and risk of complications. ${ }^{16}$ Comorbid nonpsychotic mental illness comprised depression, bipolar disorder, anxiety disorders, personality disorders, adjustment disorders and disorders of conduct and impulsivity. Substance misuse disorders comprised alcohol and drug dependence. Continuity of primary care was calculated using the Usual Provider Continuity Index, as the proportion of visits to the usual family physician or general practitioner among all visits to family physicians or general practitioners in the 2 years before the index date. Continuity was defined as high $(>80 \%)$, moderate $(51 \%$ to $80 \%)$ or low $(\leq 50 \%)$, or as infrequent use, with fewer than three visits. ${ }^{17}$

\section{Analyses}

We described general and age-specific abortion rates and abortion ratios and their 95\% confidence intervals for the 2011, 2012 and 2013 fiscal years separately. We then compared abortion rates and abortion ratios between women with and without schizophrenia by calculating rate ratios and $95 \%$ confidence intervals with Poisson regression, where women without schizophrenia were the referent group.

In the cohort of women with schizophrenia followed from 2011 to 2013, we compared the baseline characteristics of those with and without induced abortions. We then used modified Poisson regression ${ }^{18}$ to identify risk factors for induced abortion, from among age, parity, neighbourhood income quintile, region of residence, severity of schizophrenia, unstable and stable chronic medical conditions, comorbid non-psychotic mental illness and substance misuse disorders. We did not include continuity of primary care in the multivariable model because this variable may lie on the causal pathway between the other covariates and risk of induced abortion. Because of their conceptual associations with induced abortion, all other covariates were retained in the multivariable model, and a $P$-value of 0.05 was used to declare statistical significance. SAS Enterprise Guide, version 7.15 for Unix (SAS Institute, North Carolina, USA) was used for the analyses.

\section{Results}

Women with schizophrenia represented approximately $0.3 \%$ of the sample in each annual cohort (range, 0.34-0.36\%). In each year, the proportion of women without schizophrenia who had 
non-psychotic mental illness was approximately 19\% (range, 18.7$19.2 \%)$.

\section{General and age-specific abortion rates and abortion ratios}

Women with schizophrenia had a higher abortion rate than those without schizophrenia in each fiscal year under study (Table 1). This was explained by higher abortion rates in the two youngest age groups (15- to 19-year-olds and 20- to 24-year-olds), whereas abortion rates were similar between the two groups at older ages (Fig. 1). Similarly, women with schizophrenia had a higher abortion ratio than those without schizophrenia, and this finding was consistent in each fiscal year (Table 1). This was explained by higher abortion ratios among women with schizophrenia aged $\geq 20$ years, and particularly among 25 - to 39 -year-olds. There were no differences in the abortion ratios of 15- to 19-year-olds with and without schizophrenia (Fig. 2).

\section{Predictors of abortion among women with schizophrenia}

There were 11149 women with schizophrenia aged 15-44 years who were followed from 1 April 2011 to 31 March 2014. Overall, $285(2.6 \%)$ of these women had one or more induced abortions during this 3-year period. Compared with women without an induced abortion, those with an induced abortion were more likely to be $<25$ years of age and multiparous. They were less likely to have chronic medical conditions but more likely to have comorbid non-psychotic mental illness and substance misuse disorders. They were also more likely to have low continuity of primary care (Supplementary Table 1 available at https://doi.org/10.1192/ bjp.2018.262). In multivariable analyses, age $<25$ years (4.9\% (1524 years) v. 3.1\% (25-34 years); adjusted relative risk (aRR), 1.84; $95 \%$ CI $1.39-2.44)$, multiparity (3.2\% v. $2.2 \%$, aRR $2.17,95 \%$ CI 1.66-2.83), comorbid non-psychotic mental illness (2.8\% v. $1.1 \%$, aRR 2.15, 95\% CI 1.34-3.46), and substance misuse disorders, including alcohol and drug use (4.4\% v. 2.0\%, aRR 1.85, 95\% CI 1.47-2.34), were all associated with increased risk for induced abortion in the 3 years from cohort entry. Neighbourhood income quintile, region of residence, severity of schizophrenia, and stable and unstable chronic medical conditions were not associated with induced abortion risk (Table 2).

\section{Discussion}

Numerous case studies and commentaries have hypothesized that women with schizophrenia would have high induced abortion rates. ${ }^{4,5}$ We believe that ours is one of the first population-based studies, and the largest study to date, to confirm this hypothesis. Our large sample size also allowed us to examine risk for induced abortion by different outcome definitions and in specific age groups, and to identify risk factors for induced abortion. This provides new information to illuminate the types of interventions that might be appropriate in efforts to improve the sexual and reproductive health of women with schizophrenia across all age groups. Targeting high-risk groups, such as women who are younger, those who have previously given birth and those who have comorbid nonpsychotic mental illness or substance misuse disorders, may be important for efforts to ensure sexual health literacy and self-efficacy, and uptake of effective contraception.

The only other population-based studies on the risk of induced abortion among women with schizophrenia used Finnish and Danish health administrative data. ${ }^{6,7}$ Abortion rates reported in the Finnish study ${ }^{6}$ may not be directly comparable with ours because they were reported per 1000 person-years of follow-up instead of per 1000 women of reproductive age in a given year, as is more commonly used in health reports. ${ }^{3}$ Further, rather than considering the number of induced abortions per 1000 live births - the standard approach for measuring the abortion ratio - the authors calculated the proportion of all pregnancies ending in induced abortion. However, the findings are similar to ours in that a higher proportion of these pregnancies resulted in an induced abortion for women with schizophrenia than for those without. Similarly, methods used in the Danish study ${ }^{7}$ may also not be directly comparable with ours because the authors focused on the occurrence of induced abortion as the first reproductive event and because the referent group to which women with schizophrenia were compared comprised women without any mental illness (i.e. without schizophrenia, bipolar disorder, unipolar disorder or 'other' mental illness, versus without schizophrenia specifically in our study). Although abortion is legal and the cost is covered by the public health system in Finland, Denmark and Ontario, there are differences in abortion laws across these jurisdictions that may also explain the divergence in findings. In Finland and Denmark, for example, physician approval is required for abortions $>12$ weeks gestational age; such restrictions are not in place in Ontario. ${ }^{6,7}$

\begin{tabular}{|c|c|c|c|c|c|}
\hline Year & Group & $\begin{array}{l}\text { Number of } \\
\text { abortions }\end{array}$ & $\begin{array}{l}\text { Number of women/number } \\
\text { of live births }\end{array}$ & $\begin{array}{l}\text { Abortion rate/ratio } \\
(95 \% \mathrm{Cl})\end{array}$ & Rate ratio $(95 \% \mathrm{Cl})$ \\
\hline \multicolumn{6}{|c|}{$\begin{array}{l}\text { General abortion rate, per } \\
1000 \text { women }^{a}\end{array}$} \\
\hline \multirow[t]{2}{*}{2011} & Women with schizophrenia & 180 & 11149 & $16.1(14.0-18.7)$ & $1.18(1.02-1.37)$ \\
\hline & Women without schizophrenia & 44740 & 3282665 & $13.6(13.5-13.8)$ & 1.00 (referent) \\
\hline \multirow[t]{2}{*}{2012} & Women with schizophrenia & 198 & 11286 & $17.5(15.3-20.2)$ & $1.33(1.16-1.54)$ \\
\hline & Women without schizophrenia & 42642 & 3250785 & $13.1(13.0-13.2)$ & 1.00 (referent) \\
\hline \multirow[t]{2}{*}{2013} & Women with schizophrenia & 177 & 11453 & $15.5(13.3-17.9)$ & $1.20(1.04-1.39)$ \\
\hline & Women without schizophrenia & 41257 & 3212701 & $12.8(12.7-13.0)$ & 1.00 (referent) \\
\hline \multicolumn{6}{|c|}{$\begin{array}{l}\text { General abortion ratio, per } \\
1000 \text { live births }{ }^{\text {b }}\end{array}$} \\
\hline \multirow[t]{2}{*}{2011} & Women with schizophrenia & 180 & 292 & $616.4(532.7-713.4)$ & $1.81(1.56-2.09)$ \\
\hline & Women without schizophrenia & 44740 & 131321 & $340.7(337.6-343.9)$ & 1.00 (referent) \\
\hline \multirow[t]{2}{*}{2012} & Women with schizophrenia & 198 & 269 & $736.1(640.4-846.1)$ & $2.25(1.96-2.59)$ \\
\hline & Women without schizophrenia & 42642 & 130573 & $326.6(323.5-329.7)$ & 1.00 (referent) \\
\hline \multirow[t]{2}{*}{2013} & Women with schizophrenia & 177 & 299 & $592.0(510.9-685.9)$ & $1.85(1.59-2.14)$ \\
\hline & Women without schizophrenia & 41257 & 128644 & 320.7 (317.6-323.8) & 1.00 (referent) \\
\hline
\end{tabular}




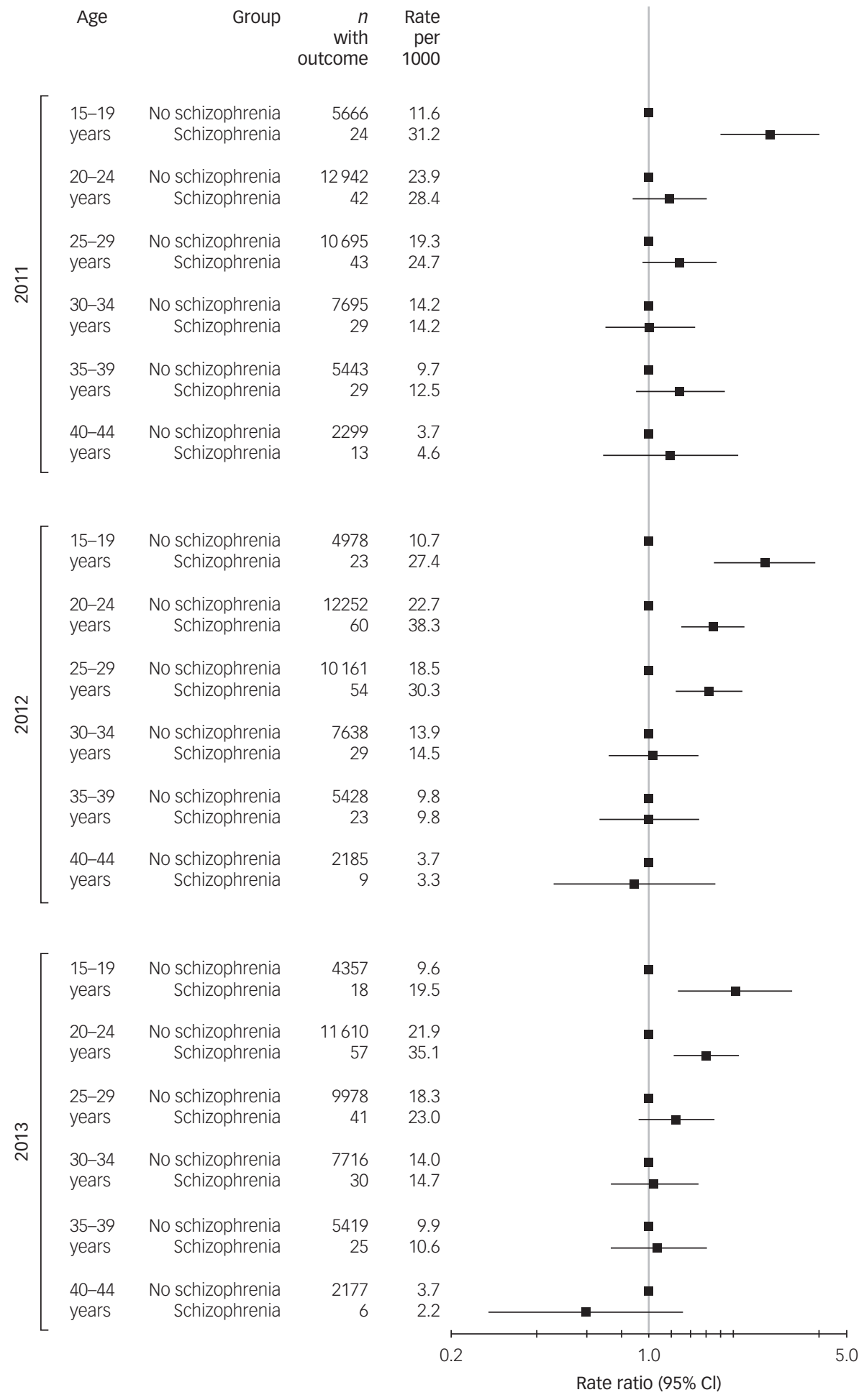

Fig. 1 Rate ratios comparing induced abortions per 1000 women of reproductive age among women with and without schizophrenia, by 5-year age groups. Data presented as number of abortions, rate per 1000 women and rate ratio compared with women without schizophrenia.

Most other studies on this topic have been small clinical studies ${ }^{4,5}$ that also suggested a high rate of induced abortion in women with schizophrenia. To our knowledge, no studies have examined risk factors for induced abortion among women with schizophrenia. Our study therefore presents novel findings that should be replicated in other jurisdictions with differing healthcare systems.

There are multiple factors that might explain elevated risk for induced abortion in women with schizophrenia. Previous studies indicate that, compared with women without schizophrenia, women with schizophrenia are less likely to use contraception and, among those who do use contraception, are at greater risk for inconsistent or improper use. ${ }^{4}$ Data suggest that women with schizophrenia experience difficulty negotiating with male partners in the use of barrier methods and other family planning issues; $40 \%$ of women with schizophrenia report discussing family planning with their partners compared with $90 \%$ of women without schizophrenia. ${ }^{19}$ Women with schizophrenia are also more likely than those without to experience sexual assault and intimate 
Age

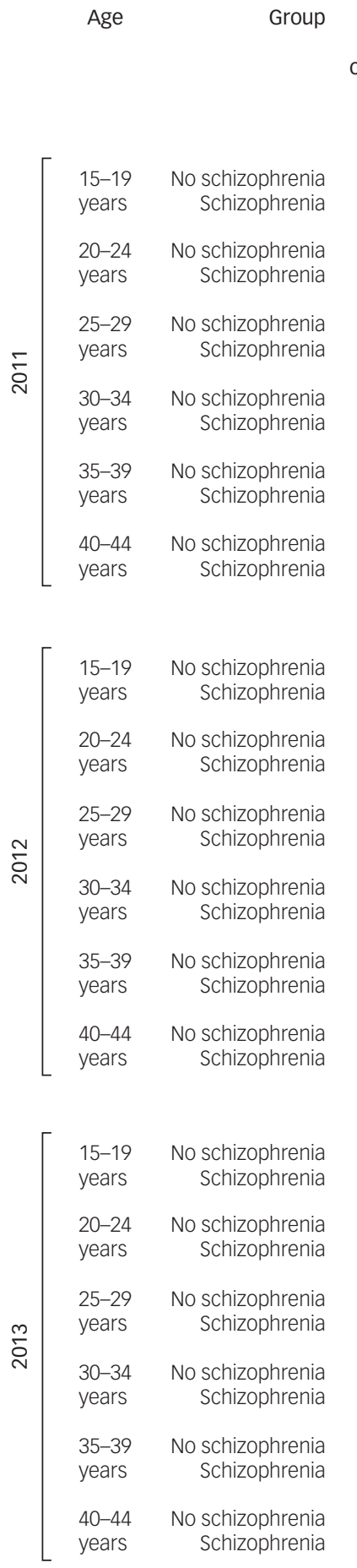

$\begin{array}{rr}n & \text { Rate } \\ \text { with } & \text { per } \\ \text { outcome } & 1000\end{array}$

$\begin{array}{rr}5666 & 1389.4 \\ 24 & 1333.3 \\ 12942 & 804.0 \\ 42 & 591.6 \\ 10695 & 290.5 \\ 43 & 573.3 \\ 7695 & 169.2 \\ 29 & 432.8 \\ 5443 & 229.1 \\ 29 & 707.3 \\ & \\ 2299 & 452.2 \\ 13 & 650.0\end{array}$

$4978 \quad 1302.8$

231642.9

$12252 \quad 804.5$

601200.0

$10161 \quad 280.1$

$\begin{array}{rr}0161 & 280.1 \\ 54 & 729.7\end{array}$

$7638 \quad 165.6$

165.6
29 439.4

$5428 \quad 226.2$

$23 \quad 418.2$

$2185 \quad 425.4$

900.0

$\begin{array}{rr}4357 & 1328.4 \\ 18 & 692.3\end{array}$

$11610 \quad 784.9$

$57 \quad 890.6$

$9978 \quad 282.7$

$41 \quad 745.5$

$7716 \quad 166.2$

$\begin{array}{ll}30 & 326.1\end{array}$

$\begin{array}{rr}5419 & 228.3 \\ 25 & 520.8\end{array}$

$2177 \quad 426.6$

426.6
428.6

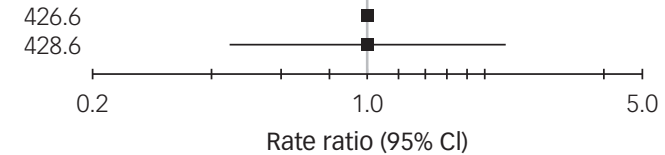

Fig. 2 Rate ratios comparing induced abortions per 1000 live births among women with and without schizophrenia, by 5-year age groups. Data presented as number of abortions, rate per 1000 live births and rate ratio compared with women without schizophrenia.

partner violence, ${ }^{20}$ and to be under the influence of drugs or alcohol during sex. ${ }^{20}$ The risk factors for induced abortion in our study, in particular the elevated risk among younger women and those with addictions, are consistent with the hypothesis that these types of vulnerability may partly explain elevated rates of unintended pregnancy and subsequent induced abortion in this population. It is also notable that comorbid non-psychotic mental illness was associated with elevated risk for induced abortion. Although one could argue that this factor may simply represent a group of women with schizophrenia with more severe illness and thus greater vulnerability to unintended pregnancy, there are other possibilities. Mood-related side-effects of oral contraception may be problematic in women already experiencing mood disturbances. ${ }^{4}$ Oral contraception can also interact with some antipsychotics (e.g. clozapine), resulting in sedation, hypotension, nausea and tremor, and with anti-epileptic drugs, which can be used as mood stabilisers in individuals with schizoaffective disorder or as adjunctive medications in treatment-resistant schizophrenia. ${ }^{4}$ Intrauterine 
Table 2 Risk factors for induced abortion in the longitudinal cohort of women with schizophrenia followed from 2011 to 2013

\begin{tabular}{|c|c|c|c|}
\hline \multirow[b]{2}{*}{ Covariate } & \multicolumn{3}{|c|}{ Women with schizophrenia $(n=11149)$} \\
\hline & $N(\%)$ with abortion & Unadjusted rate ratio $(95 \% \mathrm{Cl})$ & Adjusted relative risk $(95 \% \mathrm{Cl})$ \\
\hline \multicolumn{4}{|l|}{ Age } \\
\hline 15-24 years & $106(4.9)$ & $1.52(1.18-1.97)$ & $1.84(1.39-2.44)$ \\
\hline 25-34 years & $117(3.1)$ & Referent (1.00) & Referent (1.00) \\
\hline 35-44 years & $62(1.2)$ & $0.39(0.29-0.53)$ & $0.39(0.28-0.53)$ \\
\hline \multicolumn{4}{|l|}{ Parity } \\
\hline Multiparous & $127(3.2)$ & $1.44(1.14-1.81)$ & $2.17(1.66-2.83)$ \\
\hline Primiparous & $158(2.2)$ & Referent (1.00) & Referent (1.00) \\
\hline \multicolumn{4}{|c|}{ Neighbourhood income quintile } \\
\hline 1 (lowest) & $90(2.6)$ & $1.04(0.71-1.52)$ & $1.02(0.70-1.49)$ \\
\hline 2 & $67(2.7)$ & $1.09(0.74-1.62)$ & $1.09(0.74-1.61)$ \\
\hline 3 & $53(2.8)$ & $1.12(0.74-1.69)$ & $1.14(0.76-1.71)$ \\
\hline 4 & $35(2.0)$ & $0.82(0.52-1.29)$ & $0.84(0.53-1.31)$ \\
\hline 5 (highest) & $38(2.5)$ & Referent (1.00) & Referent (1.00) \\
\hline \multicolumn{4}{|l|}{ Residence } \\
\hline Rural & $18(2.2)$ & $0.80(0.63-1.01)$ & $0.74(0.46-1.19)$ \\
\hline Urban & $266(2.6)$ & Referent (1.00) & Referent (1.00) \\
\hline \multicolumn{4}{|c|}{ Admissions to hospital for schizophrenia } \\
\hline 0 & $211(2.6)$ & Referent (1.00) & Referent (1.00) \\
\hline 1 & $53(2.7)$ & $1.05(0.78-1.42)$ & $0.89(0.66-1.20)$ \\
\hline$\geq 2$ & $21(2.2)$ & $0.86(0.55-1.34)$ & $0.74(0.48-1.14)$ \\
\hline \multicolumn{4}{|c|}{ Stable chronic medical condition } \\
\hline Present & $101(2.2)$ & $0.75(0.58-0.96)$ & $0.97(0.76-1.25)$ \\
\hline Absent & $184(2.8)$ & Referent (1.00) & Referent (1.00) \\
\hline \multicolumn{4}{|c|}{ Unstable chronic medical condition } \\
\hline Present & $48(1.9)$ & $0.69(0.51-0.94)$ & $0.68(0.49-0.93)$ \\
\hline Absent & $237(2.8)$ & Referent (1.00) & Referent (1.00) \\
\hline \multicolumn{4}{|c|}{ Comorbid non-psychotic mental illness } \\
\hline Present & $267(2.8)$ & $2.59(1.61-4.17)$ & $2.15(1.34-3.46)$ \\
\hline Absent & $18(1.1)$ & Referent (1.00) & Referent (1.00) \\
\hline \multicolumn{4}{|c|}{ Substance misuse disorder } \\
\hline Present & $117(4.4)$ & $2.21(1.75-2.79)$ & $1.85(1.47-2.34)$ \\
\hline Absent & $168(2.0)$ & Referent (1.00) & Referent (1.00) \\
\hline
\end{tabular}

devices have been proposed as solutions for these issues in women with chronic mental illness because they are long-lasting and require little attention. However, problems with tolerability can include irregular bleeding and pain, and if sexually transmitted infections are contracted while they are in place, they are associated with risk of pelvic inflammatory disease. ${ }^{4}$

Our results suggest the need for proactive approaches to reproductive healthcare in this population, including contraception counselling. Women with schizophrenia report hesitancy raising topics related to sexual and reproductive health with their healthcare providers. ${ }^{21}$ They also report that the focus of their healthcare is often on their mental illness and that they 'become invisible as women' (Chernomas et al: pp. 1518). ${ }^{21}$ Importantly, women with serious mental illness report wanting to receive family planning services in mental health settings. ${ }^{21}$ This improves logistics by reducing the need to visit multiple clinics, which is important because many women with schizophrenia do not regularly access primary care. ${ }^{22}$ This is reflected in our data, wherein women with schizophrenia who had an abortion were more likely to have low continuity of primary care than those who did not have an abortion. Psychiatrists are uniquely positioned to address the mental illness aspect of family planning in this population, including providing training to improve psychosocial skills and assertiveness to reduce unwanted sex and giving advice about the appropriateness and clinical effect of various contraception options. Further, reproductive healthcare in women with schizophrenia requires reinforcement to prevent decay in knowledge and skills over time. If women could receive such care from a practitioner whom they see regularly, this would be ideal. ${ }^{21}$

However, there is evidence that contraception counselling and sexual health education is seldom provided to women with schizophrenia in psychiatric settings. ${ }^{23}$ Mental healthcare providers report that sexual health is more complex than other areas of physical health, they worry about reactions to what they perceive to be intrusive questions and they feel that sexual health is outside the scope of psychiatric care. ${ }^{23}$ Some may incorrectly assume that amenorrhea associated with antipsychotic treatment means loss of fertility, or that the loneliness and isolation reported by many women with schizophrenia means they are not sexually active. ${ }^{23}$ Yet, although there may be initial hesitancy among mental healthcare providers to take up reproductive health initiatives, educational interventions can greatly improve provider awareness and self-efficacy in tackling sexual health topics. ${ }^{24}$ Such educational interventions should include training on how to deal with trauma related to sexual assault and other sensitive topics. The evidence generated by our study on elevated induced abortion rates in women with schizophrenia suggests that better integration of reproduction health programming into the mental healthcare setting for women with serious mental illness may be warranted.

Finally, there are innovative models for sexual healthcare that could be explored in women with schizophrenia. Sexual health specialists have been effective in reducing unintended pregnancy in other populations, such as adolescents, and could be trained to provide outreach to populations with severe mental illness. ${ }^{25}$ Targeted preconception care models are frequently used with women with chronic medical conditions, such as diabetes, and could also be developed for women with schizophrenia to focus on mental illness management and reproductive life-planning. ${ }^{26}$ Given the scope of the issues identified in this study, a systematic plan of action to determine a framework for approaching sexual and reproductive health in this population is warranted. 


\section{Limitations}

Strengths of our study include our population-based approach, which resulted in a large, generalisable cohort of women with schizophrenia, using a validated algorithm. ${ }^{11}$ Moreover, we measured rates of induced abortion across three fiscal years, demonstrating stability in findings even for small subgroups. However, findings should be interpreted in the context of several limitations. In our cross-sectional analyses, failure to find statistically significant differences in abortion rates and abortion ratios between women with and without schizophrenia in several age-specific analyses could be explained by small numbers of induced abortions in those age groups. In our longitudinal analyses, we identified risk factors for induced abortion over a 3-year period, as opposed to lifetime risk of induced abortion. Although women could have had an induced abortion outside of our outcome window, we expect that the risk factors identified herein would be similarly associated with induced abortion risk with a longer follow-up period. We may have missed induced abortions that were paid for out of pocket or that occurred outside of Ontario, and rates of such abortions may differ by mental illness status. ${ }^{14}$ We did not have information on the timing of the induced abortion. The Finnish study suggested that terminations performed later than 12 weeks' gestational age were more common in women with schizophrenia than in those without. ${ }^{6}$ This may be because of late recognition of pregnancy, or misinterpretation or even denial of pregnancy-related symptoms. ${ }^{6}$ We also did not have information on the medical or social indication for induced abortions. However, the Finnish study suggested that most induced abortions were for social rather than medical reasons. ${ }^{6}$ We were unable to measure the effect of medication use on induced abortion risk because medication information is only available on a subset of Ontarians receiving a publicly funded drug plan. However, most well-designed studies have not shown antipsychotic medications to be associated with teratogenicity or other major perinatal health problems. There is therefore no clear indication for induced abortion for pregnancies exposed to these medications. ${ }^{27}$ Other missing contextual information included ethnicity, relationship status, occurrence of sexual assault or intimate partner violence, child protective services involvement with other children and decision-making capacity among women with schizophrenia. Finally, findings may differ in healthcare systems that do not provide universal access to medical and surgical abortion.

In conclusion, our findings are among the first populationbased estimates of induced abortion rates in women with schizophrenia and suggest avenues for future research directions and clinical response. Because of the paucity of research on this topic, our methods should be replicated in other jurisdictions with different healthcare systems, comparing with both women without schizophrenia and women with other types of mental illness. Qualitative research may also help to understand reasons for and experiences accessing abortion services in this population. High induced abortion rates in women with schizophrenia support the need for improved reproductive healthcare in this group, particularly in young women and those with comorbid non-psychotic mental illness or substance misuse disorders, who may be at greater risk for unintended pregnancy. The increased risk for induced abortion among multiparous women suggests women who have already given birth may be important targets for efforts to ensure utilisation of contraception. Better integration of reproductive healthcare in mental health settings and exploration of innovative models for sexual healthcare may facilitate contraception counselling and sexual health education in this population.
Hilary K. Brown PhD, Assistant Professor, Interdisciplinary Centre for Health and Society, University of Toronto Scarborough and Dalla Lana School of Public Health, University of Toronto and Department of Psychiatry, University of Toronto; and Adjunct Scientist, Women's College Research Institute, Women's College Hospital and Institute for Clinical Evaluative Sciences, Ontario, Canada; Cindy-Lee Dennis, PhD, Professor, Lawrence S. Bloomberg Faculty of Nursing, University of Toronto; and Scientist, Li Ka Shing Knowledge Institute, St. Michael's Hospital, Ontario, Canada; Paul Kurdyak, PhD, Sh KRP Prof Institute for Clinical Evaluative Sciences; and Psychiatrist and Director of Health Institute for Clinical Evaluative Sciences; and Psychiatrist and Director of Health
Outcomes and Performance Evaluation (HOPE), Centre for Addiction and Mental Health, Ontario, Canada; Simone N. Vigod, MSC, MD, FRCPC, Associate Professor, Department of Psychiatry, University of Toronto; and Scientist, Women's College Research Institute, Women's College Hospital and Institute for Clinical Evaluative Sciences, Ontario, Canada

Correspondence: Hilary K. Brown, Interdisciplinary Centre for Health \& Society, University of Toronto Scarborough, 1265 Military Trail, Toronto, ON M1C 1A4, Canada. Email: hk.brown@utoronto.ca

First received 25 Apr 2018, final revision 17 Sep 2018, accepted 17 Oct 2018

\section{Supplementary material}

Supplementary material is available online at https://doi.org/10.1192/bjp.2018.262

\section{Funding}

This study was supported by the Institute for Clinical Evaluative Sciences (ICES), which is funded by an annual grant from the Ontario Ministry of Health and Long-Term Care. The opinions, results and conclusions reported in this paper are those of the authors and are independent from the funding sources. No endorsement by ICES or the Government of Ontario is intended or should be inferred. Parts of this material are based on data and information compiled and provided by the Canadian Institute for Health information (CHI). HOwever, the andyses, conclusions, opinions and statements expressed herein are those of the author and not necessarily those of $\mathrm{ClHI}$.

\section{Acknowledgements}

We gratefully acknowledge the province of Ontario, Canada for their support of this study through their research grants programme.

\section{References}

1 Andreasen NC. Symptoms, signs, and diagnosis of schizophrenia. Lancet 1995: 346: $477-81$.

2 Vigod SN, Seeman MV, Ray JG, Anderson GM, Dennis CL, Grigoriadis S, et al. Temporal trends in general and age-specific fertility rates among women with schizophrenia (1996-2009): a population-based study in Ontario. Canada. Schizophrenia Res 2012; 139: 169-75.

3 Jatlaoui TC, Shah J, Mandel MG, Krashin JW, Suchdev DB, Jamieson DJ, et al. Abortion surveillance-United States, 2014. MMWR Surveill Summ 2017; 66 : $1-48$.

4 Matevosyan NR. Reproductive health in women with serious mental illnesses: a review. Sex Disabil 2009; 27: 109-18.

5 Ozcan NK, Boyacioglu NE, Enginkaya S, Dinc $\mathrm{H}$, Bilgin $\mathrm{H}$. Reproductive health in women with serious mental illnesses. J Clin Nurs 2014; 23: 1283-91.

6 Simoila L, Isometsa E, Gissler M, Suvisaari J, Sailas E, Halmesmaki E, et al. Schizophrenia and induced abortions: a national register-based follow-up study among Finnish women born between 1965-1980 with schizophrenia and schizoaffective disorder. Schizophrenia Res 2018; 192: 142-7.

7 Laursen TM, Munk-Olsen T. Reproductive patterns in psychotic patients. Schizophrenia Res 2010; 121: 234-40.

8 World Health Organization (WHO). International statistical classification of diseases and related health problems. WHO, 1994.

9 American Psychiatric Association (APA). Diagnostic and statistical manual of mental disorders. 4th ed. APA, 2000.

10 Williams Jl, Young WA. Summary of studies on the quality of health care administrative databases in Canada. In Patterns of Health Care in Ontario, The ICES Practice Atlas (eds V Goel, JI Williams, GM Anderson, P Blackstien-Hirsch, C Fooks and CD Naylor): 339-45. Canadian Medical Association, 1996.

11 Kurdyak P, Lin E, Green D, Vigod S. Validation of a population-based algorithm to detect chronic psychotic illness. Can J Psychiatry 2015; 60: 362-8.

12 Pope MA, Joober R, Malla AK. Diagnostic stability of first-episode psychotic disorders and persistence of comorbid psychiatric disorders over 1 year. Can $J$ Psychiatry 2013; 58: 588-94. 
13 Chamberlain A, Rauh J, Passer A, McGrath M, Burket R. Issues in fertility control for mentally retarded female adolescents: I. Sexual activity, sexual abuse, and contraception. Pediatrics 1984; 73: 445-50.

14 Urquia ML, Moineddin R, Jha P, O'Campo PJ, McKenzie K, Glazier RH, et al. Sex ratios at birth after induced abortion. CMAJ 2016; 188(9): E181-90.

15 Canadian Institute for Health Information. Important Notes Regarding Coverage. Canadian Institute for Health Information, 2014 (https://www.cihi. ca/en/ta_11_alldatatables20130221_en.pdf).

16 Johns Hopkins Bloomberg School of Public Health. The Johns Hopkins ACG® System, version 9.0. Johns Hopkins University, Maryland, USA, 2009 (https:// www.hopkinsacg.org/)

17 Jee SH, Cabana MD. Indices for continuity of care: a systematic review of the literature. Med Care Res Rev 2006; 63: 158-88.

18 Zou GY. A modified Poisson regression approach to prospective studies with binary data. Am J Epidemiol 2004; 159: 702-6.

19 Pehlivanoglu K, Tanriover O, Tomruk N, Karamustafalioglu N, Oztekin E and Alpay N. Family planning needs and contraceptive use in female psychiatric outpatients. Turkish J Fam Med Primary Care 2007; 1: 32-5.

20 Kelly DL, Conley RR. Sexuality and schizophrenia: a review. Schizophrenia Bull 2004; 30: 767-79.

21 Chernomas WM, Clarke DE, Chisholm FA. Perspectives of women living with schizophrenia. Psychiatr Serv 2000; 51: 1517-21.

22 Copeland LA, Zeber JE, Wang CP, Parchman ML, Lawrence VA, Valenstein $\mathrm{M}$, et al. Patterns of primary care and mortality among patients with schizophrenia or diabetes: a cluster analysis approach to the retrospective study of healthcare utilization. BMC Health Serv Res 2009; 9: 127

23 Hugues E, Edmondson AJ, Onyekwe I, Quinn C, Nolan F. Identifying and addressing sexual health in serious mental illness: views of mental health staff working in two National Health Service organizations in England. Int J Ment Health Nurs 2018; 27(3): 966-74.

24 Quinn C, Happell B, Welch A. The 5-As framework for including sexual concerns in mental health nursing practice. Issues Ment Health Nurs 2013; 34 $17-24$

25 Hayter M. Reaching marginalized young people through sexual health nursing outreach clinics: evaluating service use and the views of service users. Nursing Open 2005; 22(4): 339-46.

26 Wahabi HA, Alzeidan RA, Bawazeer GA, Alansari LA, Esmaeil SA. Preconception care for diabetic women for improving maternal and fetal outcomes: a systematic review and meta-analysis. BMC Pregnancy Childbirth 2010; 10: 63

27 Vigod SN, Gomes T, Wilton AS, Taylor VH, Ray JG. Antipsychotic drug use in pregnancy: high dimensional, propensity matched, population-based cohort study. BMJ 2015; 350: h2298. 